\title{
PRESERVACIÓN DE LA VEJIGA EN EL CÁNCER DE RECTO CON INVASIÓN DE LA PRÓSTATA
}

\author{
F. HERRANZ AMO*, F. MUÑOZ JIMÉNEZ**, D. SUBIRÁ RÍOS*, M. GÓMEZ ESPÍ***, \\ F.A. CALVO MANUEL***
}

*Servicio de Urología. **Unidad de Cirugía Colo-Rectal. ***Servicio de Oncología Radioterápica. Hospital General Universitario Gregorio Marañón. Madrid.

Actas Urol Esp. 28 (6): 447-451, 2004

\section{RESUMEN}

PRESERVACIÓN DE LA VEJIGA EN EL CÁNCER DE RECTO CON INVASIÓN DE LA PRÓSTATA

El cáncer colo-rectal es el 2ㅇ tumor en frecuencia en Europa. En el 5-10\% de los casos existe infiltración de órganos urológicos. Cuando la infiltración afecta a la vejiga o a la próstata, la exenteración pelviana anterior es el tratamiento con el que se consiguen mayor porcentaje de márgenes quirúrgicos libres de tumor y mayores probabilidades de supervivencia a los 5 años. En casos muy seleccionados de infiltración prostática es posible preservar la vejiga, realizando una prostatectomía y amputación abdominoperineal en bloque cumpliendo con los requisitos quirúrgicos oncológicos y mejorando de forma importante la calidad de vida del paciente.

Debido a la escasa bibliografía existente (dos artículos con 3 casos) presentamos nuestra experiencia en 2 pacientes a los que se les realizó radioquimioterapia preoperatoria citoreductora, prostatectomía y amputación abdominoperineal en bloque y radioterapia intraoperatoria. Describimos la técnica quirúrgica utilizada, que presenta diferencias sustanciales con la prostatectomía estándar y precisa de una buena coordinación entre el equipo de cirujanos y el de urólogos.

PALABRAS CLAVE: Carcinoma colo-rectal. Invasión prostática. Invasión vesículas seminales. Prostatectomía.

\section{ABSTRACT \\ PRESERVATION OF THE BLADDER IN RECTAL CANCER WITH PROSTATIC INVASION}

Colorectal cancer in the $2^{\text {nd }}$ commonest cancer in Europe. In 5-10\% of cases there is infiltration of urological organs. When infiltration affects the bladder or the prostate, anterior pelvic exenteration is the treatment that achieves the largest percentage of tumor free margins and the best 5-year survival. In very select cases of prostatic infiltration, the bladder can be preserved and prostatectomy and abdominoperineal block resection are carried out fulfilling oncological surgical requirements and producing an important improvement in the patient's quality of life.

Owing to the very scarse published literature (two articles with three cases) we contribute our experience of 2 patients who received cytoreducing preoperative radiochemotherapy, prostatectomy and block abdominoperineal amputation and intraoperative radiotherapy. We describe the surgical technique used, which is substantially different from standard prostatectomy and requires good coordination between surgeons and urologists.

KEY WORDS: Colorectal carcinoma. Prostatic invasion. Seminal vesicle invasion. Prostatectomy. 
$\mathrm{E}^{1}$ cáncer colo-rectal supone el $15 \%$ de los diagnósticos tumorales y la segunda causa de muerte por cáncer en USA ${ }^{1}$. En Europa representa el $12 \%$ de los tumores en el hombre y el $14 \%$ en las mujeres, la estimación de incidencia para 1995 fue de 49,6 casos por 100.000 en el hombre y de 33,9 por 100.000 en la mujer, siendo la segunda causa tumoral en incidencia en ambos sexos ${ }^{2}$.

El tratamiento de elección del carcinoma de recto es la resección quirúrgica con excisión mesorrectal total (TME) y amplios márgenes laterales. En ocasiones, es necesario la resección total o parcial de órganos adyacentes afectados para intentar conseguir márgenes quirúrgicos libres de tumor. Aproximadamente existe infiltración de órganos urológicos en el 5-10\% de los pacientes, aumentando hasta el 50\% en los carcinomas $\mathrm{T} 4^{3}$. La vejiga, la próstata y las vesículas seminales son los órganos urológicos más frecuentemente afectados. El empleo de radioquimioterapia preoperatoria en dosis de 45-50 Gy (potenciada con fluopirimidinas) disminuye el tamaño tumoral, facilitando la resección de tumores localmente avanzados, permitiendo en ocasiones preservar el aparato esfinteriano, controlar micrometástasis existentes en el meso del recto evitando la diseminación tumoral y disminuye la recidiva local aumentando la supervivencia. El empleo de radioterapia intraoperatoria (RIO) dirigida a la zona del lecho tumoral una vez extirpado el mismo, intensifica la RT externa preoperatoria promoviendo el control pélvico en casos de exéresis con márgenes estrechos o con riesgo de residuo microscópico.

En los pacientes con infiltración de la próstata el tratamiento estándar es la exenteración pelviana anterior, lo que implica una derivación urinaria con una morbi-mortalidad no despreciable. En ocasiones se realiza una resección parcial de la próstata en el tiempo perineal de la proctosigmoidectomía, pero estudios multicéntricos han demostrado que la supervivencia a los 5 años es muy superior cuando se realiza la multiresección visceral en bloque, $52 \%$ frente al $17 \% \%^{4,5}$.

Cuando el cáncer de recto invade exclusivamente la próstata o las vesículas seminales la realización de una prostatectomía con preservación vesical es posible en casos muy selecciona- dos. Debido a la escasa experiencia publicada, dos artículos ${ }^{6,7}$ y sólo 3 pacientes, comunicamos nuestra experiencia.

\section{CASOS CLÍNICOS}

\section{Caso $n^{-} 1$}

Paciente de 48 años con antecedentes de nefrectomía izquierda por tuberculosis y colecistectomía por litiasis. Consulta por dolor anal y rectorragia. Tacto rectal: se palpa una masa exofítica que ocupa toda la circunferencia rectal. Colonoscopia: tumoración nodular ulcerada próxima a los márgenes del ano. Biopsia: adenocarcinoma grado II. Ecografia endorectal: lesión circunferencial ulcero-vegetante desde canal anal a $4 \mathrm{~cm}$. de margen anal que infiltra la vesícula seminal derecha y rompe la cápsula prostática con adenopatías mayores de $8 \mathrm{~mm}$. En la TC no se aprecian metástasis a distancia. Se realiza tratamiento citoreductor preoperatorio con quimioradioterapia según protocolo de nuestro Centro $^{8}$, recibiendo Tegafur (5-fluoruracilo) oral y una dosis total de radioterapia de 45 Gy (fraccionamiento convencional). En enero/2000 se realiza amputación abdominoperineal y prostatectomía en bloque, en el mismo acto quirúrgico se aplica radioterapia intraoperatoria administrándose 12,50 Gy. La anatomía patológica de la pieza corresponde a un adenocarcinoma que invade la grasa perirectal, la próstata y afecta a 8 ganglios linfáticos sin afectación de los márgenes de resección quirúrgicos. $\mathrm{El}$ paciente evoluciona con íleo prolongado, infección de la herida quirúrgica y fuga persistente de contraste por la anastomosis vesico-uretral que se resolvió con sondaje vesical prolongado. En febrero/2000 inicia quimioterapia adyuvante (esquema 5- fluoruracilo/leucovorin) recibiendo 3 ciclos y suspendiéndose por TVP de miembro inferior izquierdo. El paciente presenta incontinencia de orina a grandes esfuerzos y disfunción eréctil. En mayo/2002 el paciente está asintomático con marcadores tumorales negativos presentando masa presacra en el TC, clínica y morfológicamente estable.

\section{Caso $n^{\circ} 2$}

Paciente de 54 años sin antecedentes de interés que consultó por episodio de rectorragia. Tacto rectal: lesión de más de $2 \mathrm{~cm}$ de diámetro 
inmediatamente por encima del margen anal que ocupa la hemicircunferencia derecha. Colonoscopia: lesión neoplásica adyacente a margen anal. Biopsia: adenocarcinoma grado II. Ecografía endorectal: lesión que invade próstata en su extremo más inferior, no adenopatías. En TC no metástasis a distancia. Se realiza tratamiento citorreductor preoperatorio con quimioradioterapia según protocolo de nuestro Centro ${ }^{8}$, recibiendo Tegafur oral y una dosis total de radioterapia de 45 Gy (fraccionamiento convencional). En diciembre/2001 se realiza amputación abdominoperineal y prostatectomía en bloque, en el mismo acto quirúrgico se aplica radioterapia intraoperatoria (RIO) administrándose 12,50 Gy. La anatomía patológica de la pieza corresponde a un adenocarcinoma que invade la grasa perirrectal sin invasión de la próstata y sin afectación de los márgenes de resección quirúrgicos. Evolución post-operatoria sin incidencias. En febrero/2001 inicia quimioterapia adyuvante (esquema 5- fluoruracilo/leucovorin). El paciente presenta incontinencia de orina a grandes esfuerzos y disfunción eréctil que se trata mediante inyecciones intracavernosas de alprostadil.

\section{TÉCNICA QUIRÚRGICA}

Con el paciente en posición de litotomía y a través de una laparotomía media supra e infraumbilical se accede a la cavidad intraperitoneal, una vez comprobada la ausencia de metástasis locales o a distancia; se procede a la liberación del colon sigmoide y descendente, a la ligadura de la arteria mesentérica inferior en su origen, de la vena en el borde inferior pancreático y a la transección del colon a unos $10 \mathrm{~cm}$ de la reflexión peritoneal. Liberación del recto de la cara posterior de la vejiga hasta las vesículas seminales. El equipo de cirujanos inicia el tiempo perineal y el equipo urológico se incorpora al campo abdominal. Apertura del espacio de Retzius, incisión bilateral de la fascia endopélvica y sección de los ligamentos puboprostáticos. Ligadura y sección del complejo venoso dorsal. Disección del ápex prostático y la uretra membranosa, el dedo índice del urólogo situado entre la uretra y el recto sirve de referencia al equipo perineal para completar la disección anterior del recto. Sección de la uretra membranosa.
Apertura de la cara anterior y posterior del cuello vesical, disección de la cara anterior de ambas vesículas seminales y sección de los deferentes a nivel de la ampolla deferencial según la técnica de Campbell ${ }^{9}$. Disección, ligadura y sección de ambos pedículos prostáticos superiores, pero sin disecar la cara posterior de las vesículas seminales (Fig. 1). El equipo de cirujanos completa la disección posterior y lateral del recto incluyendo el meso con la fascia visceral íntegra, extrayéndose la pieza quirúrgica en bloque (Fig. 2) por la incisión perineal. Reconstrucción del cuello vesical "en raqueta" y anastomosis vesico-uretral utilizando la maniobra de Gil-Vernet ${ }^{10}$ para la intraabdominalización de la uretra membranosa, en el primer caso se realizó vía abdominal y en el segundo caso vía perineal y comprobación de la sutura de la cara anterior por vía abdominal. En el segundo caso se aproximaron los fascículos del músculo elevador del ano para darle una base de sustentación a la uretra y sutura de la cara anterior de la vejiga a la sínfisis del pubis para impedir la incarceración de la misma en la fosa rectal según describen Klee y Grmoljez ${ }^{11}$. Colocación simulada del aplicador de RIO biselado de mayor

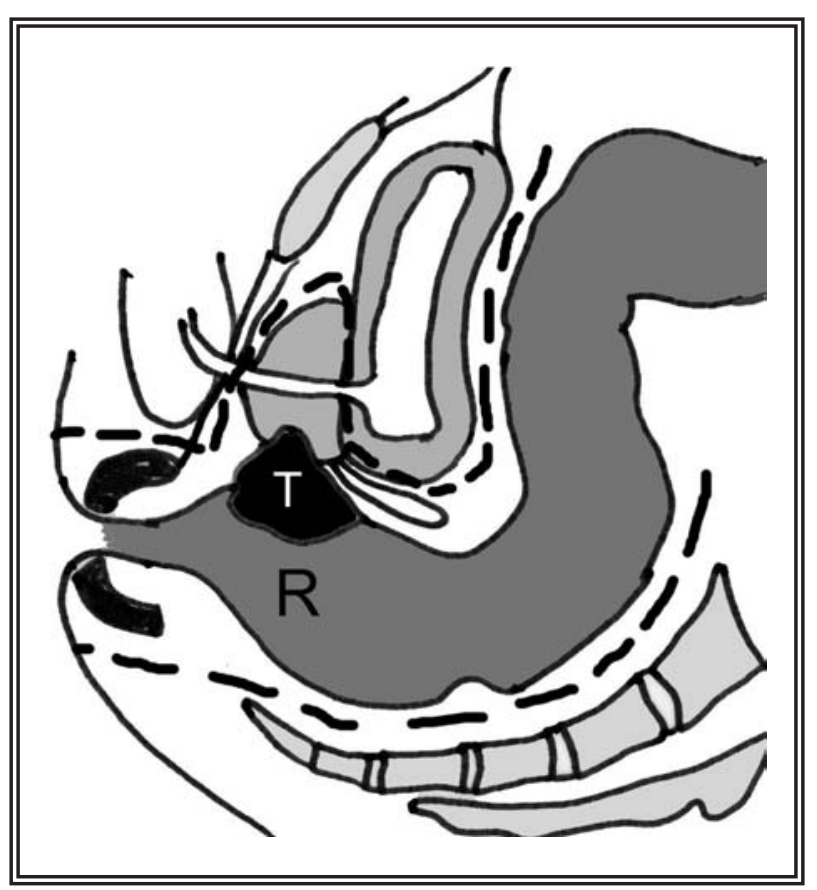

FIGURA 1. Esquema de un corte sagital de la pelvis en el hombre. T: tumor en la cara anterior del recto. $R$ : recto. Linea discontinua - márgenes de resección de la pieza formada por recto-sigma, próstata y vesiculas seminales. 


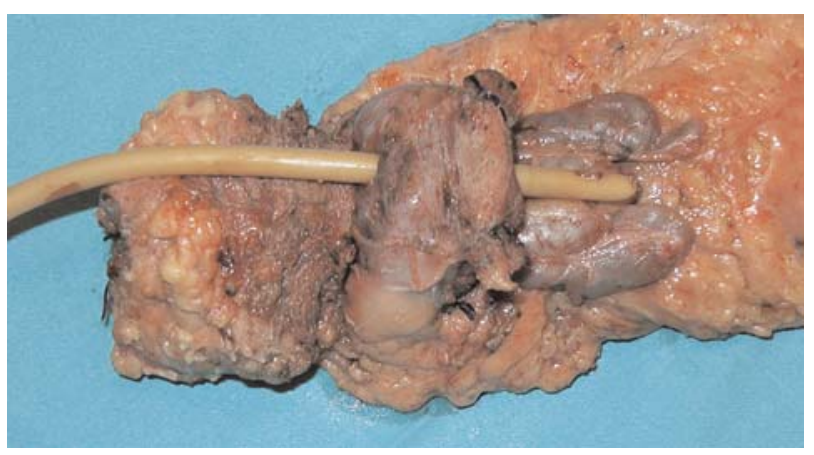

FIGURA 2. Pieza quirúrgica donde se puede observar el esfinter anal, la próstata con las vesiculas seminales y el recto.

diámetro posible en la pelvis menor vacía de asas intestinales, evitando que los uréteres queden expuestos en el campo de irradiación, para conseguir la máxima cobertura de la región presacra. Empaquetamiento de la cavidad abdominal y transporte del paciente con un equipo de ventilación y monitorización anestésica portátil a la sala del acelerador lineal para someterle a una irradiación con electrones de alta energía. De nuevo en el quirófano se realiza revisión de la cavidad abdominal, montaje de la colostomía definitiva, colocación de drenajes y cierre de las heridas quirúrgicas.

\section{DISCUSIÓN}

El paciente con sospecha de invasión de órganos urológicos por un cáncer colo-rectal debe de ser intervenido por un equipo quirúrgico multidisciplinar, formado por cirujanos especializados en cirugía colo-rectal y urólogos con formación oncológica $^{12}$. El objetivo debe de ser la resección en bloque de los órganos afectados para obtener márgenes quirúrgicos libres de afectación tumoral y evitar la diseminación tumoral en la cavidad abdominal.

Cuando el cáncer rectal afecta a los órganos urológicos pelvianos la exenteración pelviana implica la realización, al menos, de una derivación urinaria. Pudiendo realizarse una sustitución vesical con intestino ${ }^{13}$ o una derivación urinaria externa, cuando se realice una colostomía definitiva es posible la realización de una derivación doble (heces y orina) en un único estoma ${ }^{14}$. En casos muy seleccionados con afectación de la próstata o las vesículas seminales es posible pre- servar la vejiga ${ }^{5,6}$ evitando la derivación urinaria, disminuyendo las complicaciones post-operatorias y mejorando la calidad de vida del paciente.

La administración de radio-quimioterapia preoperatoria en el cáncer de recto localmente avanzado disminuye el riesgo de recidiva local ${ }^{15} \mathrm{y}$ puede facilitar la cirugía de preservación esfinteriana. Del Valle y cols. ${ }^{16}$ han demostrado en un subgrupo de pacientes con afectación tumoral entre 4 y $8 \mathrm{~cm}$ del margen anal, que aumenta de forma significativa la cirugía de preservación esfinteriana en la Unidad de Cirugía Colo-Rectal de nuestro Hospital. Con el esquema terapéutico utilizado en nuestro Centro el $94 \%$ de los pacientes están libres de recidiva local a los cuatro años de seguimiento ${ }^{8}$.

Ganem y cols. ${ }^{17}$ realizan la prostatectomía perineal cuando es necesario la resección total del recto. Campbell y cols. ${ }^{6}$ describen por primera vez la técnica de prostatectomía y rectosigmoidectomía en bloque con preservación rectal por vía abdominal en dos pacientes que cursan con disfunción eréctil y un control miccional satisfactorio. Fujisawa y cols. ${ }^{7}$ comunican un caso de amputación abdominoperineal con prostatectomía en bloque evolucionando el paciente con disfunción eréctil, continencia urinaria diurna e incontinencia nocturna. Klee y Grmoljez ${ }^{11}$ comunican en 3 pacientes con cáncer de próstata y cáncer de recto la posibilidad de realizar en un mismo tiempo, pero no en bloque, una prostatectomía radical y una resección anterior baja de recto o amputación abdominoperineal, recomendando la aproximación de los músculos elevadores del ano por detrás de la anastomosis vésicouretral y la fijación de la cara anterior de la vejiga al pubis para evitar su incarceración en la fosa rectal cuando se realice amputación abdominoperineal.

La obtención de márgenes quirúrgicos negativos y la ausencia de infiltración ganglionar son los dos factores pronósticos más importantes en la cirugía del cáncer colo-rectal avanzado. Stief y cols. analizan la supervivencia en pacientes con márgenes quirúrgicos y ganglios negativos con sospecha de afectación vesical o prostática, encuentran una mayor supervivencia en los pacientes con resección total del órgano urológico que en los que se realizó resección parcial ${ }^{3}$, ya 
que la distinción intraoperatoria entre infiltración tumoral o adhesión inflamatoria es imposible de discernir. Aunque en su serie sólo el $52 \%$ de los órganos resecados tenía infiltración tumoral, Stief y cols. ${ }^{12}$ recomiendan, ante la sospecha de afectación de órganos urológicos, la resección total del mismo; ya que consideran que la separación de ellos en el acto quirúrgico disminuye de forma muy importante la supervivencia del paciente, siendo más importante la radicalidad que la preservación de la función vesical.

En pacientes muy seleccionados con cáncer de recto y afectación únicamente de la próstata o vesículas seminales, la prostatectomía y rectosigmoidectomía en bloque con preservación de la vejiga es una técnica que consigue márgenes quirúrgicos libres y mejora la calidad de vida para estos pacientes frente a la exenteración pelviana anterior.

\section{REFERENCIAS}

1. American Cancer Society. Facts and figures, special sections: colorectal cancer. 2002. http:/www.cancer.org/statistics/.

2. BRAY F, SANKILA R, FERLAY J, PARKIN DM.: Estimates of cancer incidence and mortality in Europe in 1995. Eur $J$ Cancer 2002; 38: 99-166.

3. STIEF CG, JONAS U, RAAB R.: Long-term follow-up after surgery for advanced colorectal carcinoma involving the urogenital tract. Eur Urol 2002; 41: 546-550.

4. SUGARBAKER PH, CORLEW S.: Influence of surgical techniques on survival in patients with colorectal cancer. Dis Colon Rectum 1982; 25: 545-547.

5. GALL FP, TONAK J, ALTENDORF A.: Multivisceral resections in colorectal cancer. Dis Colo Rectum 1987; 30: 337-341.

6. CAMPBEL SC, CHURCH JM, FAZIO VW, KLEIN EA, PONTES JE.: Surg Gynecol Obstet 1993, 176: 605608.

7. FUJISAWA M, UENO K, KAMIDONO S.: Novel bladder sparing surgery for select patients with advanced rectal carcinoma. J Urol 2002; 167: 643-644.
8. CALVO FA, GÓMEZ ESPÍ, DÍAZ GONZÁLEZ JA y cols.: Intraoperative presacral electron boost following preoperative chemoradiation in $\mathrm{T} 3-4, \mathrm{Nx}$ rectal cancer: initial local effects and clinical outcome analysis. Radiother Oncology 2002; 62: 201-206.

9. CAMPBELL EW.: Total prostatectomy with preliminary ligation of the vascular pedicles. J Urol 1959; 81: 464-467.

10. GIL-VERNET JM, GUTIÉRREZ DEL POZO R, CARRETERO P, CAMPOS JA, GIL-VERNET JM Jr.: Urogenital diaphragm raising maneuver. $J$ Urol 1988; 140: 555-558.

11. KLEE LW, GRMOLJEZ P.: Combined radical retropubic prostatectomy and rectal resection. Urology 1999; 54: 679-681.

12. STIEF CG, RAAB R.: Interdisciplinary abdominourological surgery for advanced colorectal carcinoma involving the urogenital tract. BJU International 2002; 89: 496-503.

13. SKINNER DG, SHERROD A.: Total pelvic exenteration with simultaneous bowel and urinary reconstruction. J Urol 1990; 144: 1433-1438.

14. CARTER MF, DALTON DP, GARNET JE.: Simultaneous diversion of the urinary and fecal streams utilizing a single abdominal stoma: the doble-barreled wet colostomy. J Urol 1989; 141: 1189-1191.

15. KAAKFOOR BM, WILLET CG, SHELLITO PC, KAUFMAN DS, DALY WJ.: The impact of 5Fluorouracil and intraoperative electron beam radiation therapy on the outcome of patients with locally advanced primary rectal and rectosigmoid cancer. Ann Surg 1998; 228: 194-200.

16. DEL VALLE E, MUÑOZ F, CALVO FA y cols.: Preoperative chemoradiation and sphincter preservation in rectal cancer. 2nd International Symposium Sphincter Saving Treatment in rectal Cancer. Lyon, 2001.

17. GANEM PJ, WALLWORK DW, JOLLIFFE L, KAY J.: Rectal carcinoma invading the prostate gland. Arch Surg 1957; 75: 85-89.

Dr. F. Herranz Amo

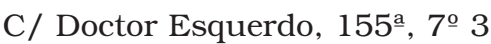

28007 Madrid

(Trabajo recibido el 16 diciembre de 2003) 